\title{
The Tragedy of the Commons in a Violent World
}

\author{
Petros G. Sekeris ${ }^{1}$
}

\footnotetext{
${ }^{1}$ Department of Economics \& Finance, University of Portsmouth. E-mail: petros.sekeris@port.ac.uk. This project is the result of long discussions and problem-solving sessions with Giacomo De Luca to whom I am highly indebted. The manuscript has been substantially improved thanks to the insights of two anonymous referees of this journal. I am thankful to Mark Armstrong, the editor of this journal, for his support, and to Jean-Marie Baland, David Comerford, Joan Esteban, Michelle Garfinkel, Marco Giani, Francois Libois, Paul Maarek, Christophe Muller, Paul Reding, Steven Salant, Santiago Snchez Pges, Raphael Soubeyran, Tanguy Van Ypperselle, and Patrick Warren for for their helpful comments and support.
} 


\begin{abstract}
Earlier research has shown that the tragedy of the commons may be resolved by Folk theorems for dynamic games. In this article we graft on a standard naturalresource exploitation game the possibility to appropriate the resource through violent means. Because conflict emerges endogenously as resources get depleted, the threat supporting the cooperative outcome is no longer subgame perfect, and thus credible. The unique equilibrium is such that players exploit non-cooperatively the resource when it is abundant and they revert to conflict when it becomes scarce. The players' utility is shown to be lower even if conflict wastes no resources.

Keywords: Tragedy of the Commons, Conflict, Dynamic Game JEL Classification: C73, D74, Q2
\end{abstract}




\section{Introduction}

A vast body of literature has studied the problem of the management of common pool resources. This literature tends to assume away notions such as violent appropriation and conflict. Yet the historical example of Easter Island's collapse demonstrates the close connections between the management of the commons and violence. When the first European expedition reached Easter Island in 1722, its inhabitants were living in a heavily depleted environment. According to Jared Diamond (2005) the society was organized in hierarchical clans that peacefully competed with each other for power supremacy by erecting stone statues. For this purpose, the island's tallest trees were cut down, as a result of which a rapid deforestation occurred. ${ }^{1}$ The exhaustion of this vital natural resource used to construct fishing canoes spurred an acceleration of the island's inland fauna exploitation, eventually creating food scarcities so severe that cannibalism emerged. In 1680, as the situation must have reached dramatic levels of deprivation of all kinds, and with the elites proving unable to deliver their promises to their people, a sort of military coup occurred, followed by a prolonged period of wars.

The Easter Islanders are but one example of the inability of a group of individuals to manage the common pool resources in an efficient way, therefore being the victims of the tragedy of the commons (Hardin 1968). Yet, such failure to cooperate in the resource exploitation may seem at odds with existing theories on the subject. The essence of the problem at stake is that for the economically efficient management

\footnotetext{
${ }^{1} \mathrm{~A}$ controversy on the real causes of the Island's deforestation is still open among scientists (Hunt and Lipo 2011).
} 
rule to emerge spontaneously it is necessary that property rights over the resource be well defined and enforced (Coase 1960, Hardin 1968). The required institutions are, however, absent in many settings because of a lack of local (sub-national) or global (international) governance. Ostrom (1990) and Baland and Platteau (1996) narrate how local communities may succeed in overcoming the tragedy of the commons. Yet, the mechanisms identified by these authors necessitate a strong form of social capital that is absent in many CPR contexts. Extending Folk theorems for repeated games to dynamic games, economists then proposed a more general solution to the CPR management inefficiency. Cooperation on the efficient exploitation of a depletable resource may be achieved by the threat of reverting to non-cooperation in case of noncompliance to the agreed behavior (Cave 1987, Dutta 1995, Sorger 2005, Dutta and Radner 2009). ${ }^{2}$ Why should therefore the efficient outcome fail to be implemented in dynamic settings? Consistently with the Easter Island example, the theory developed in this article identifies the anticipation of conflict as the causal element for the breakdown of cooperation.

In this article we introduce the possibility of violently appropriating the stock of resources in a dynamic game of CPR exploitation. The main finding of the article is that when resources are dynamically depleted along the cooperative extraction path, conflict will occur with certainty when resources are sufficiently depleted. When the resource becomes scarce, should the players decide to fight over the remaining stock of resources, they will be acquiring few armaments. Consequently, conflicts would then be of low intensity, which would also mean that they would be less

\footnotetext{
${ }^{2}$ For an early work on the strategic exploitation of a CPR in a dynamic setting, see Levhari and Mirman (1980).
} 
destructive. As a consequence, in the presence of depleted stocks of resources, players may violently appropriate at a low cost part of the goods which they will then manage privately. Given that the first best solution fails to be stable, no alternative path of play to the conflict-reversion strategy can be an equilibrium either. As a consequence, the punishment threat for not respecting a cooperative agreement stops being credible (i.e. subgame perfect), thus implying that cooperation itself breaks down. Interestingly, even if conflict intensity is extremely low at equilibrium, the resulting breakdown of cooperation implies that the players' utility will always be lower as compared to a conflict-free setting. This follows from the over-exploitation of the resource in the periods where the stock is sufficiently abundant for players not to fight over its control.

This article contributes to the expanding field of conflict theory. Whereas the initial writings mainly focused on static properties of conflict models, the dynamic dimension has received increased attention lately. Some interesting issues that have been explored are the timing of conflict when the players' strength evolves either exogenously (Bester and Konrad 2004), or endogenously yet in a deterministic way (Powell 2012), deterrence and preventive motives for conflict (Jackson and Morelli 2009, Chassang and Padró i Miquel 2009), as well as dynamic incentives to pursue fighting over time (Leventoglu and Slantchev 2007). A common dynamic incentive in conflict settings is to attempt modifying the sharing of the contested pie in the short run through violent means in order to enhance one's continuation value, although the more valuable the prize, the more intense and thus costly will the conflict be (McBride and Skaperdas 2007, Esteban et al. 2011). 
Garfinkel (1990) and Yared (2010) both investigate the scope for reaching peaceful agreements in repeated games of resource exploitation and conflict. Garfinkel (1990) was the first to study Folk theorems for conflict models and established that in a repeated prisoner-dilemma type of setting, peace can be supported as an equilibrium for sufficiently patient players. More recently, Yared (2010) extended the analysis to a context involving information imperfections and considered the harshest existing subgame perfect punishments. In Yared's model, because of informational imperfections, temporary punishments under the form of non-permanent wars may occur along the equilibrium path. Common to these two articles is the repeated nature of the game, which implies that the resource at stake is a constant flow of wealth. Such models are well suited therefore to formalize potential conflicts over the control of economic sectors and territories. In our setting, the resource is instead conceptualized as a stock that regenerates at some non-negative rate, thus implying that our model captures better the dynamics of finite resources, whether they regenerate or not.

Acemoglu, Golosov, Tsyvinski, and Yared (AGTY, 2012) is perhaps the closest contribution to ours as they consider a dynamic game of trade and conflict over an exhaustible resource whose value is a function of its scarcity. In exploring the conditions favoring the peaceful dynamic trade as opposed to an invasion by a resourceless player, AGTY show that if the depletable resource, which is located in one country, is exploited competitively, individual firms fail to internalize the negative externality of their individual extraction on the increased likelihood of foreign intervention. The high prices that this exploitation generates in the future, boosts the future incen- 
tives for conflict, thus feeding back in the firms' short run incentives to exploit the resource, which eventually triggers immediate conflict. Whereas both our models are dynamic and feature an exhaustible resource, they differ along several dimensions.

A first dimension along which these two articles differ is that whereas in AGTY the players are either resource users able to wield power if needed to appropriate the resources, or else resource exploiters, in the present work players are both exploiters and consumers of the resource. This modeling assumption comes at a cost given that, to keep the model tractable, in this article, unlike in AGTY, the players are assumed to be symmetric. Second, we impose log-utility functions that in AGTY would translate in war-incentives not depending on the amount of resources. Instead, in our framework arming is not a separable cost, hence implying that conflict generates a double inefficiency: it destroys part of the stock of resources, and it diverts otherwise productive resources to fighting activities, which further contributes to the depletion of the stock. Hence, whereas in AGTY the cost of conflict and its outcome are both exogenous (assailant wins with unit probability), we instead enable both sides to decide their armament levels, hence also making the winning probabilities endogenous. These modeling differences underlie the differences in the mechanics conducive to conflict in AGTY and in this article. In AGTY conflict results from the market price of resources being an increasing price of their scarcity, whereas the cost of violently appropriating the resources through war remains constant. In our framework instead, as resources become scarce, the investments in armaments decrease as well, thus reducing the burden of the conflict and equally making it less destructive. Lastly, we extend the analysis to the concept of subgame perfection 
instead of focusing on the subset of Markov perfect equilibria.

In the next section we develop a standard benchmark model of dynamic resource exploitation and we identify sufficient conditions for the efficient solution to be sustained as an equilibrium. In Section 3 we introduce the possibility of reverting to violence in this same game. Lastly, Section 4 concludes.

\section{The model: peaceful world}

We consider an infinite time horizon game of a renewable resource exploitation. Time is discrete and denoted by $t=0,1, \ldots, \infty$. Two players labeled 1 and 2 simultaneously decide at each time period the amount of resources to exploit from a common pool of renewable resources. In time zero the world is endowed with a stock of $r_{0}$ resources that grows at a linear rate $\gamma \cdot{ }^{3}$ Players costlessly invest effort in resource-use, so that player $i$ 's appropriation effort of renewable resources in time $t$ is denoted by $e_{i, t}$, with $e_{i, t} \in[0, \bar{e}], \bar{e}>r_{0}$. Player $i$ 's associated consumption is given by $x_{i, t}$ such that:

$$
x_{i, t}= \begin{cases}e_{i, t} & \text { if } e_{1, t}+e_{2, t} \leq r_{t} \\ \frac{e_{i, t}}{e_{1, t}+e_{2, t}} r_{t} & \text { otherwise }\end{cases}
$$

The resources available in period $t, r_{t}$, equal:

$$
r_{t}=(1+\gamma)\left(r_{t-1}-x_{1, t-1}-x_{2, t-1}\right)
$$

\footnotetext{
${ }^{3}$ The growth rate of the resources could equally be capturing the (constant) growth of productivity resulting from technological advances.
} 
The instantaneous utility of any player $i$ in time $t$ is given by:

$$
u_{i, t}=\ln \left(x_{i, t}\right)
$$

And the discounted life-time utility of player $i$ in time period 0 equals:

$$
U_{i, 0}=\sum_{t=0}^{\infty} \delta^{t} \ln \left(x_{i t}\right)
$$

Where $\delta$ stands for the discount rate.

We define a strategy for player $i$ as $e_{i}=\left\{e_{i, t}\right\}_{t=0}^{\infty}$.

\section{The efficient solution}

The efficient solution of this resource exploitation game is given by the solution to the central planner's following problem:

$$
\begin{aligned}
& \max _{e_{1}, e_{2}} \sum_{i=1,2} \sum_{t=0}^{\infty} \delta^{t} \ln \left(x_{i, t}\right) \\
& \text { s.t. } \quad(1) \text { and (2) }
\end{aligned}
$$

We denote by $V^{c}\left(r_{t}\right)$ the value function of this problem given the resource stock $r_{t}$, meaning that the indirect aggregate utility can be expressed as a Bellman equation:

$$
V^{c}\left(r_{t}\right)=\arg \max _{e_{1, t}, e_{2, t}}\left[\sum_{i=1,2} \ln \left(x_{i, t}\right)+\delta V^{c}\left(r_{t+1}\right)\right]
$$

Next, given the assumed regeneration rule, the above expression can be written as: 


$$
V^{c}\left(r_{t}\right)=\arg \max _{e_{1, t}, e_{2, t}}\left[\sum_{i=1,2} \ln \left(x_{i, t}\right)+\delta V^{c}\left((1+\gamma)\left(r_{t}-x_{1, t}-x_{2, t}\right)\right)\right]
$$

Differentiating (6) with respect to the two decision variables, $e_{1, t}$ and $e_{2, t}$, and making use of (1) we obtain the following system of equations:

$$
\left\{\begin{array}{l}
\frac{\partial V^{c}\left(r_{t}\right)}{\partial e_{1, t}}=\frac{1}{x_{1}^{c}\left(r_{t}\right)}-\delta(1+\gamma) \sum_{i=1,2} V_{i}^{c^{\prime}}\left((1+\gamma)\left(r_{t}-x_{1}^{c}\left(r_{t}\right)-x_{2}^{c}\left(r_{t}\right)\right)\right)=0 \\
\frac{\partial V^{c}\left(r_{t}\right)}{\partial e_{2, t}}=\frac{1}{x_{2}^{c}\left(r_{t}\right)}-\delta(1+\gamma) \sum_{i=1,2} V_{i}^{c^{\prime}}\left((1+\gamma)\left(r_{t}-x_{1}^{c}\left(r_{t}\right)-x_{2}^{c}\left(r_{t}\right)\right)\right)=0
\end{array}\right.
$$

Where these equations hold because the constraint $e_{1, t}+e_{2, t} \leq r_{t}$ will never be binding, as $\lim _{r_{t} \rightarrow 0} V_{i}^{c^{\prime}}=+\infty$.

From (7) we deduce that $x_{1}^{c}\left(r_{t}\right)=x_{2}^{c}\left(r_{t}\right)=x^{c}\left(r_{t}\right)$. To derive the efficient equilibrium, we inquire whether $x^{c}\left(r_{t}\right)$ may be a linear function of its argument so that $x^{c}\left(r_{t}\right)=\lambda^{c} r_{t}$. This assumption implies that the stock of resources in time period $t+1$ can be expressed as $r_{t+1}=(1+\gamma)\left(1-2 \lambda^{c}\right) r_{t}$. Replacing in equation (4), together with using the regeneration rule gives us:

$V^{c}\left(r_{t}\right)=2\left[\ln \left(\lambda^{c} r_{t}\right)+\delta \ln \left(\lambda^{c}(1+\gamma)\left(1-2 \lambda^{c}\right) r_{t}\right)+\delta^{2} \ln \left(\lambda^{c}(1+\gamma)^{2}\left(1-2 \lambda^{c}\right)^{2} r_{t}\right)+\ldots\right]$

Rearranging the terms of (8) gives us:

$$
V^{c}\left(r_{t}\right)=\frac{2 \ln \left(\lambda^{c} r_{t}\right)}{1-\delta}+2 \sum_{\tau=0}^{\infty} \delta^{\tau} \ln \left((1+\gamma)^{\tau}\left(1-2 \lambda^{c}\right)^{\tau}\right)
$$

Thus implying that $V^{c^{\prime}}\left(r_{t}\right)=\frac{2}{(1-\delta) r_{t}}$. Substituting in (7) for $V^{c^{\prime}}($.$) yields:$ 


$$
\frac{1}{\lambda^{c} r_{t}}-\frac{2 \delta(1+\gamma)}{(1-\delta)(1+\gamma)\left(1-2 \lambda^{c}\right) r_{t}} \Rightarrow \lambda^{c}=\frac{1-\delta}{2}
$$

This last expression stands for the share of available resources consumed by each player under the efficient solution. The proportion of the stock of resources which is preserved from one period to another therefore equals $(1+\gamma) \delta .{ }^{4}$ The optimal consumption for any individual $i$ is therefore given by:

$$
x_{t}^{c}=\frac{(1-\delta) r_{t}}{2}
$$

Hence implying that any player's life time utility in time $t$ can be written as:

$$
V_{i, t}^{c}=\frac{1}{1-\delta}\left[\ln \left(\frac{(1-\delta) r_{t}}{2}\right)+\frac{\delta}{1-\delta} \ln (\delta(1+\gamma))\right]
$$

Expression (11) gives us the life time utility of players in what we term the "cooperative scenario".

For this first-best solution to be a subgame perfect equilibrium (SPE), it is necessary that no profitable deviation exists for either player. The instantaneous utility players obtain by deviating from the efficient solution can straightforwardly be shown to be higher than the instantaneous utility of cooperating. Hence, for the first-best solution to be a SPE, it needs to be sustained by some form of decentralized dynamic punishment. Any such threat ought to be subgame perfect itself, however. In what follows we consider the harshest subgame perfect punishments.

\footnotetext{
${ }^{4}$ Notice that the resource is dynamically depleted if $(1+\gamma) \delta<1 \Leftrightarrow \gamma<\frac{1-\delta}{\delta}$. For the problem to be salient, in the remainder of the article we assume that this condition is satisfied.
} 


\section{Harshest punishment}

We denote by $\mathcal{H}$ the set of strategies generating the harshest punishments of this game. At time $\tau$ any strategies satisfying the following conditions belong to $\mathcal{H}$.

$$
e_{i, \tau} \geq r_{\tau}, e_{i, \tau+t} \in[0, \bar{e}] \quad \forall t>0, \forall i \in\{1,2\}
$$

As there exists no profitable unilateral deviation from such strategy profiles, they describe a SPE where the players' associated utility is infinitely negative. From this stems the following result:

Proposition 1. The first-best solution to the resource exploitation game is always supported as a subgame perfect equilibrium.

Proof. Consider the following punishment: if $\left(e_{1, t}, e_{2, t}\right) \neq\left(e_{1 t}^{c}, e_{2, t}^{c}\right)$ for some $t$, then $e_{t+1} \in \mathcal{H}$. Given that $V_{i, t}$ reported in equation (11) is finite, deviating and obtaining infinitely negative utility can never be profitable.

Because of the logarithmic specification we have chosen, the harshest punishments consist in fully depleting the resource. Given that the players' utility would then become infinitely negative, any path of play generating some non-infinitely negative payoff to both players may be sustained as a SPE. By extension therefore, the efficient solution is equally supported as a an equilibrium. The full-depletion SPE supporting the efficient solution do not survive stronger equilibrium refinements (as the extensive form trembling hand perfect equilibrium). It can be shown, however, that there exist milder punishments sustaining the efficient solutions for some $1>\delta>\bar{\delta}>0$. As 
our theory will be shown to be true for any subgame perfect equilibrium, we do not further explore these punishments.

\section{The model: violent world}

We now introduce in the game the possibility for players to revert to violence to establish private property rights over the common pool resource.

The players begin by unilaterally deciding whether or not to initiate a contest over the establishment of property rights on the common pool resource. Conflict ensues if either or both players opt for conflict. In case of indifference, we assume that players refrain from initiating conflict. In a second stage, players simultaneously decide the amount of effort to devote to resource extraction to be used for building weapons $\left(\hat{e}_{1, t}, \hat{e}_{2, t}\right)$. Player $i$ 's associated amount of weapons acquired in $t$ is given by $g_{i, t}$ such that:

$$
g_{i, t}=\left\{\begin{array}{cc}
\hat{e}_{i, t} \quad \text { if } & \hat{e}_{1, t}+\hat{e}_{2, t} \leq r_{t} \\
\frac{\hat{e}_{i, t}}{\hat{e}_{1, t}+\hat{e}_{2, t}} r_{t} & \text { otherwise }
\end{array}\right.
$$

If conflict occurs in time $t$, a share $\varphi\left(g_{1, t}, g_{2, t}\right)$ of the remaining stock of resources is destroyed, and player $i$ eventually retains control over a share $p\left(g_{i, t}, g_{j, t} ; \alpha\right)=$ $\frac{g_{i, t}+\alpha / 2}{g_{i, t}+g_{j, t}+\alpha}$ of the remaining stock of resources. The parameter $\alpha$ gauges the difficulty of modifying that sharing when entering into an armed confrontation. We make the following assumptions on the function $\varphi\left(g_{i, t}, g_{j, t}\right)$ :

$$
\varphi_{g_{i}}, \varphi_{g_{j}} \geq 0 \text { if } \varphi\left(g_{i}, g_{j}\right)<1, \varphi_{g_{i}}, \varphi_{g_{j}}=0 \text { otherwise } ; \varphi_{g_{i}}(0,0)=\varphi_{g_{j}}(0,0)=0
$$


$\varphi_{g_{i} g_{i}}, \varphi_{g_{j} g_{j}}, \varphi_{g_{i} g_{j}} \geq 0$

We are therefore assuming that war is increasingly destructive for higher (individual or aggregate) levels of the strength of the contestants involved in the conflict. ${ }^{5}$

Lastly, in stage 3, players decide the extraction efforts $e_{1, t}$ and $e_{2, t}$ for producing consumables given that the pool is either commonly owned if no conflict took place in period $t$ or before, or else it is partitioned into private properties.

If conflict is decided in time period $\tau$, player 1's utility equals:

$U_{1, \tau}^{w}=\frac{\ln \left((1-\delta) p\left(g_{1, \tau}, g_{2, \tau} ; \alpha\right)\left(1-\varphi\left(g_{1, \tau}, g_{2, \tau}\right)\right)\left(r_{\tau}-\sum_{i=\{1,2\}} g_{i, \tau}\right)\right)}{1-\delta}+\frac{\delta \ln ((1+\gamma) \delta)}{(1-\delta)^{2}}(13)$

After conflict occurred period $\tau$ player $i$ becomes the sole manager of a share $p\left(g_{1, \tau}, g_{2, \tau} ; \alpha\right)\left(1-\varphi\left(g_{1, \tau}, g_{2, \tau}\right)\right)$ of the stock of resources $r_{\tau}$, out of which $g_{1, \tau}+g_{2, \tau}$ have been extracted for building weapons. As the players will afterwards manage the resource efficiently, it is easy to show using the previous section's techniques that the optimal extraction rates over their private properties will then be $(1-\delta)$.

The timing of the resource extraction game in a violent world at each point in time is the following:

1. Players simultaneously decide whether to initiate conflict

2. Players simultaneously decide the amount of consumables to dedicate to weaponbuilding $\left(g_{1, t}, g_{2, t}\right)$

\footnotetext{
${ }^{5}$ These assumptions are sufficient for deriving the results in this article. The (weak) convexity of $\varphi(.,$.$) guarantees the monotonicity of the players' optimal weapons as a function of the stock of$ resources. Sufficiently concave functions would lead to non-monotonic relationships.
} 
3. Players simultaneously decide their extraction efforts $\left(e_{1, t}, e_{2, t}\right)$ for consumption purposes $\left(x_{1, t}, x_{2, t}\right)$

\section{Optimization in a violent world}

Optimizing (13) for player 1 with respect to $g_{1, \tau}$ subject to (12), and simplifying we obtain the following F.O.C.:

$$
\begin{array}{r}
\frac{g_{2, \tau}+\frac{\alpha}{2}}{\left(g_{1, \tau}+g_{2, \tau}+\alpha\right)^{2}}\left(1-\varphi\left(g_{1, \tau}, g_{2, \tau}\right)\right)\left(r_{\tau}-g_{1, \tau}-g_{2, \tau}\right) \\
-p\left(g_{1, \tau}, g_{2, \tau} ; \alpha\right) \varphi_{g_{1, \tau}}\left(g_{1, \tau}, g_{2, \tau}\right)\left(r_{\tau}-g_{1, \tau}-g_{2, \tau}\right)-p\left(g_{1, \tau}, g_{2, \tau} ; \alpha\right)\left(1-\varphi\left(g_{1, \tau}, g_{2, \tau}\right)\right)=0(14)
\end{array}
$$

Re-arranging, simplifying, and dropping the time subscripts for notational reasons, we obtain:

$$
\begin{array}{r}
\left(g_{2}+\frac{\alpha}{2}\right)\left(1-\varphi\left(g_{1}, g_{2}\right)\right)\left(r-g_{1}-g_{2}\right)-\varphi_{g_{1}}\left(g_{1}, g_{2}\right)\left(r-g_{1}-g_{2}\right)\left(g_{1}+\frac{\alpha}{2}\right)\left(g_{1}+g_{2}+\alpha\right) \\
-\left(1-\varphi\left(g_{1}, g_{2}\right)\right)\left(g_{1}+\frac{\alpha}{2}\right)\left(g_{1}+g_{2}+\alpha\right)=0(15
\end{array}
$$

In the first subsection of the Appendix, we show that the S.O.C. is satisfied at optimality, so that the objective function is quasi-concave in $g_{1}$.

Using the equivalent expression for player 2 implies that at optimality the following equality must hold:

$$
\frac{g_{2}+\frac{\alpha}{2}}{\left(g_{1}+\frac{\alpha}{2}\right)\left(g_{1}+g_{2}+\alpha\right)}\left(1-\varphi\left(g_{1}, g_{2}\right)\right)-\varphi_{g_{1}}\left(g_{1}, g_{2}\right)=\frac{g_{1}+\frac{\alpha}{2}}{\left(g_{2}+\frac{\alpha}{2}\right)\left(g_{1}+g_{2}+\alpha\right)}\left(1-\varphi\left(g_{1}, g_{2}\right)\right)-\varphi_{g_{2}}\left(g_{1}, g_{2}\right)
$$


From this last expression we deduce that $g_{1}^{*}=g_{2}^{*}$, for otherwise the equality is necessarily violated. ${ }^{6}$ We can therefore implicitly express the optimal amount of weapons $g_{1, \tau}^{*}=g_{2, \tau}^{*}=g_{\tau}^{*}$ :

$$
\left(1-\varphi\left(g_{\tau}^{*}, g_{\tau}^{*}\right)\right)\left(r_{\tau}-4 g_{\tau}^{*}-\alpha\right)-\left(2 g_{\tau}^{*}+\alpha\right) \varphi_{g_{\tau}^{*}}\left(r_{\tau}-2 g_{\tau}^{*}\right)=0
$$

Where $\varphi_{g_{\tau}^{*}}$ stands for a short notation of $\varphi_{g}\left(g^{*}\left(r_{\tau}\right), g^{*}\left(r_{\tau}\right)\right)$. The utility of player $i$ in time period $\tau$ thus equals:

$$
V_{1, \tau}^{w}=\frac{\ln \left(\frac{(1-\delta)}{2}\left(1-\varphi\left(g_{\tau}^{*}, g_{\tau}^{*}\right)\right)\left(r_{\tau}-2 g_{\tau}^{*}\right)\right)}{1-\delta}+\frac{\delta \ln ((1+\gamma) \delta)}{(1-\delta)^{2}}
$$

The implicit description of $g_{\tau}^{*}$ as given by (16) allows us to deduce the following result:

Lemma 1. Militarization increases monotonically in the stock of resources. Moreover, for any finite $r$, if $\alpha>\bar{\alpha}(r)$, conflicts are weaponless.

The intuition of Lemma 1 is straightforward. The first part is a standard result in the conflict literature: the larger the prize at stake, the more effort the contestants will invest in attempting to grab the resource. The second part of the lemma tells us that the smaller the ability of the contestants to influence the partition of the resource by holding weapons, the smaller will be their investments, with conflict eventually being weaponless for high values of $\alpha$. For the remaining of the article we assume that $\alpha>\bar{\alpha}(0)$ so that for very low levels of resources, conflicts are weaponless

\footnotetext{
${ }^{6}$ If, for instance, $g_{1}>g_{2}$, both terms of the LHS are individually smaller than their counterpart of the RHS.
} 
because the players do not find it optimal to attempt influencing the de facto sharing of the resources.

Lemma 1 is very useful in showing the following lemma:

Lemma 2. For sufficiently low levels of resources, the efficient extraction rate cannot be the equilibrium extraction rate.

For the proof, see the third subsection of the Appendix.

This lemma constitutes an important building block of our main result because it implies that if the punishment inflicted on deviators from the efficient agreement was the reversion to conflict, then the efficient solution would not be an equilibrium outcome for low levels of resources. The intuition behind this result lies in the increasingly small cost of conflict when the resources are sufficiently depleted: as the value of the prize at stake diminishes the players will extract less resources for weapon-building if conflict was to occur, hence implying that the damage generated by conflict will equally be contained. This implies that players eventually prefer to free-ride on their opponent's cooperative effort in the short run and then to revert to a low-intensity conflict. For sufficiently low stocks of resources, therefore, the short run benefits of reneging on the cooperative behavior coupled with low conflict intensity, and thus low dynamic inefficiencies from destroyed production, outmatch the dynamic foregone future consumption from eternal cooperation.

In Section 2 we characterized the set of SPE constituting the harshest subgame perfect punishments, and equally mentioned the existence of alternative SPE that could support the cooperative solution as an equilibrium. In light of these strategies, we need to determine whether in a conflictual world cooperation is still sustainable 
at equilibrium. By the very definition of the efficient solution, it is not Paretodominated, hence implying that compared to any potential alternative SPE at least one player is strictly better-off under the efficient solution. The very fact that both players find it optimal to deviate from the cooperative strategy and then to revert to conflict when $r$ becomes sufficiently small equally implies therefore that no alternative SPE can exist.

A relevant question is whether the cooperative equilibrium whereby both players extract the quantities determined by the social planner on the equilibrium path may still be implemented in the short run. We could, for instance, devise a strategy where players would credibly threat each other to revert to non-cooperation for a finite number of periods in case of non-compliance, before eventually declaring conflict. The following Proposition establishes that this is never the case:

Proposition 2. In a depletable resource exploitation game where players can revert to violence to appropriate the common pool resource, the equilibrium is such that players exploit non-cooperatively the resource when the stock of resources is abundant, whereas they revert to conflict if the resource becomes sufficiently scarce.

For the proof see the fourth subsection of the Appendix.

Proposition 2 highlights the important implications of introducing conflict in a dynamic game of CPR management. Interestingly, the prospect of conflict makes off-the-equilibrium-path threats non credible, when these same threats would have supported the first-best outcome in the absence of conflict. Thus, conflict may be expected not to occur for a long period of time, but the very expectation of conflict induces the players not to cooperate. Because the conflict decision is endogenous, 
when opting for conflict the (symmetric) players necessarily fare better than by exploiting the resources non-cooperatively. This implies that the unique (Paretodominated) non-cooperative equilibrium of this game ceases being an equilibrium with the introduction of conflict. Yet, although conflict removes from the game its worst equilibria, it equally eliminates the Pareto-superior ones as the cooperative equilibrium can no longer be sustained. It is therefore noteworthy to emphasize that irrespectively of the amount of weapons invested in the - possibly distant - conflict, the following corollary holds true:

Corollary 1. The anticipation of conflict accelerates resource depletion as compared to a cooperative path of play, and reduces the players' equilibrium utility.

Proof. This follows from three facts. Assume conflict takes place in time $t$. Then, ( $i$ ) from that time period on the players behave similarly to the cooperative scenario given that they privately own part of the resources. Hence for the same amount of resources $r_{t}$, conservation would be equivalent under either scenario. Next, (ii) in period $t$, under conflict resources are devoted to building weapons, and conflict destroys part of the stock of resources. These two forces reduce the post-conflict stock of resources as compared to the cooperative path of play. Lastly, (iii) in any period preceding conflict, under the conflict scenario the players play non-cooperatively, hence depleting faster the stock of resources as compared to the cooperative path of play.

This finding must not be mis-interpreted as conflict reducing the players' utility because of either the opportunity cost of conflict, or the direct inefficiencies tied 
to fighting activities. The reduction in the players' utility is primarily linked to the inability to sustain the cooperative Pareto-superior equilibrium, because the punishments supporting this equilibrium are no longer credible.

\section{Concluding remarks}

We introduced in a standard dynamic game of common pool resource management the possibility of privatizing the common resource by reverting to conflict. If conflict is waged in the presence of high stocks of resources, the players invest important amounts in conflict. The high opportunity cost of this operation coupled with the potentially destructive nature of heavily armed conflicts induces the players to refrain

from initiating conflict. For low levels of resources, however, conflict becomes a profitable option. The implications of this finding are profound because the strategies that are traditionally used to sustain cooperation are no longer subgame perfect. As a consequence, the cooperative equilibrium breaks down. In the presence of abundant resources, the players exploit non-cooperatively the $\mathrm{CPR}$ in expectation of conflict occurring after the stock has been sufficiently depleted at some point in the future. Importantly, compared to the cooperative equilibrium, the unique equilibrium in this CPR game with conflict involves a faster depletion of the resource, and lower utility levels for the players. This is true even when the players expend minimal resources in conflict.

The theory developed in this article assumes away the existence of a third party able to enforce peaceful Pareto-superior agreements. It is crucial to keep in mind that 
it is in such contexts that the "tragedy of the commons" emerges. Indeed, Coase's theorem teaches us that if property rights are well defined and enforced in the absence of transaction costs, then the first best solution is implemented. In the presence of strong institutions efficient solutions are always implementable, and in the absence of transaction costs there always exist transfers between players leaving everyone better-off. Interestingly in such settings, whether ownership is private or public would make no difference in terms of efficiency. The "tragedy of the commons" is at play in the presence of weak institutions. Yet, it is precisely in such contexts that the theory developed in this article becomes relevant. Our theory therefore underlines the importance of improving institutions when attempting to achieve more efficient economic outcomes.

In this article we have assumed a perfect information setting. Lifting this hypothesis and endowing a third-party with private information over the stock of resources or its regeneration rate would enable the third party to potentially improve the outcome's efficiency by strategically selecting the information to transmit, even in the presence of weak institutions. This interesting question is a fruitful topic to explore in future research. 


\section{Appendix}

This Appendix contains the proofs of the lemmas and propositions. It also demonstrates the quasi-concavity of the utility function under conflict.

\subsection{Quasi-concavity of $U_{1, t}^{w}\left(g_{1}\right)$}

Proof. By implicitly computing $\partial^{2} U_{1, t}^{w}\left(g_{1}\right) / \partial g_{1} \partial g_{1}$ using equation (15), we obtain:

$$
\begin{array}{r}
\frac{\partial^{2} U_{1, t}^{w}\left(g_{1}\right)}{\partial g_{1} \partial g_{1}}=-\left(g_{2}+\frac{\alpha}{2}\right) \varphi_{g_{1}}\left(r-g_{1}-g_{2}\right)-\left(2 g_{1}+g_{2}+\frac{3 \alpha}{2}\right) \varphi_{g_{1}}\left(r-g_{1}-g_{2}\right) \\
-\varphi_{g_{1} g_{1}}\left(r-g_{1}-g_{2}\right)\left(g_{1}+\frac{\alpha}{2}\right)\left(g_{1}+g_{2}+\alpha\right)+2 \varphi_{g_{1}}\left(g_{1}+\frac{\alpha}{2}\right)\left(g_{1}+g_{2}+\alpha\right) \\
-2\left(g_{1}+g_{2}+\alpha\right)(1-\varphi)(
\end{array}
$$

The only positive term in this expression is $2 \varphi_{g_{1}}\left(g_{1}+\frac{\alpha}{2}\right)\left(g_{1}+g_{2}+\alpha\right)$. Yet, by equation (15) we deduce that $2 \varphi_{g_{1}}\left(g_{1}+\frac{\alpha}{2}\right)\left(g_{1}+g_{2}+\alpha\right)<\left(2 g_{2}+\alpha\right)(1-\varphi)$, and it thus follows that the sign of the above expression is negative.

\subsection{Proof of Lemma 1}

Proof. Applying the implicit functions' theorem on expression (16) we obtain:

$$
\frac{\partial g^{*}}{\partial r}=-\frac{(1-\varphi)-\varphi_{g^{*}}\left(2 g^{*}+\alpha\right)}{-2 \phi_{g^{*}}\left(r_{\tau}-4 g^{*}-\alpha\right)-4(1-\phi)-2 \phi_{g^{*}}\left(r_{\tau}-2 g^{*}\right)+2\left(2 g^{*}+\alpha\right) \phi_{g^{*}}-\left(2 g^{*}+\alpha\right) \phi_{g^{*} g^{*}}\left(r_{\tau}-2 g^{*}\right)}
$$

From (16) we deduce that $(1-\varphi)>\varphi_{g^{*}}\left(2 g^{*}+\alpha\right)$. As a consequence, the numerator of (A-2) is positive, whereas the denominator is negative, hence making the whole 
expression strictly positive for any value of $r$. Next, because the first term of (14) tends to zero when $\alpha$ becomes arbitrarily big, it follows that for any finite $r$ there exists a value $\bar{\alpha}(r)$ such that, $g^{*}(\bar{\alpha}(r), r)=0$ for any $\alpha \geq \bar{\alpha}(r)$, and $g^{*}(\bar{\alpha}(r), r)>0$ for any $\alpha<\bar{\alpha}(r)$.

\subsection{Proof of Lemma 2}

Proof. Our aim is to demonstrate that $\exists \underline{r}>0$ such that $\forall r_{t}<\underline{r}$

$$
\ln \left(x_{1}^{d e v}\left(x_{2}^{c}\left(r_{t}\right)\right)\right)+\delta V_{1, t+1}^{w}\left(r_{t+1}\right)>V_{1, t}^{c}\left(r_{t}\right)
$$

Replacing by the optimal values of the variables we have already derived, this expression can be re-written as:

$$
\begin{aligned}
& \ln (\left.x_{1}^{\operatorname{dev}}\left(\frac{1-\delta}{2} r_{t}\right)\right)+\frac{\delta \ln \left(\frac{(1-\delta)}{2}\left(1-\varphi\left(g_{t+1}^{*}, g_{t+1}^{*}\right)\right)\left(r_{t+1}-2 g_{t+1}^{*}\right)\right)}{1-\delta} \\
& \quad+\frac{\delta^{2} \ln ((1+\gamma) \delta)}{(1-\delta)^{2}}>\frac{1}{1-\delta}\left[\ln \left(\frac{(1-\delta) r_{t}}{2}\right)+\frac{\delta}{1-\delta} \ln (\delta(1+\gamma))\right]
\end{aligned}
$$

We proceed is two steps.

Assume first that in some time $t, \varphi()=$.0 and $\varphi_{g}=0$. Moreover, take $\underline{r}$ sufficiently small such that $\alpha \geq \bar{\alpha}(\underline{r})$. If that was the case, we would necessarily have $g_{t}^{*}=0$. Replacing in (17) would then yield:

$$
V_{1, t+1}^{w}=\frac{1}{1-\delta} \ln \left(\frac{(1-\delta) r_{t+1}}{2}\right)+\frac{\delta}{(1-\delta)^{2}} \ln ((1+\gamma) \delta)
$$


Replacing in (A-4) would give us:

$$
\begin{array}{r}
\ln \left(x_{1}^{\text {dev }}\left(\frac{1-\delta}{2} r_{t}\right)\right)+\frac{\delta \ln \left(\frac{(1-\delta)}{2} r_{t+1}\right)}{1-\delta}+\frac{\delta^{2} \ln ((1+\gamma) \delta)}{(1-\delta)^{2}} \\
>\frac{1}{1-\delta}\left[\ln \left(\frac{(1-\delta) r_{t}}{2}\right)+\frac{\delta}{1-\delta} \ln (\delta(1+\gamma))\right]
\end{array}
$$

Optimizing the LHS of this inequality w.r.t. $x_{1}^{\text {dev }}\left(x_{2}^{c}\right)$ we obtain the next F.O.C.:

$$
\frac{1}{x_{1}^{d e v}}=\frac{\delta}{1-\delta} \frac{1}{(1+\delta) r_{t} / 2-x_{1}^{d e v}}
$$

Solving for $x_{1}^{d e v}$ gives us:

$$
x_{1}^{\text {dev }}\left(x_{2}^{c} ; r_{t}\right)=\frac{(1-\delta)(1+\delta)}{2} r_{t}
$$

Substituting in (A-4) gives the following expression:

$$
\begin{aligned}
\ln \left(\frac{(1-\delta)(1+\delta)}{2} r_{t}\right) & +\frac{\delta \ln \left(\frac{(1-\delta)}{2}\left(\frac{(1+\gamma) \delta(1+\delta)}{2} r_{t}\right)\right)}{1-\delta}+\frac{\delta^{2} \ln ((1+\gamma) \delta)}{(1-\delta)^{2}} \\
& >\frac{1}{1-\delta}\left[\ln \left(\frac{(1-\delta) r_{t}}{2}\right)+\frac{\delta}{1-\delta} \ln (\delta(1+\gamma))\right]
\end{aligned}
$$

Simplifying yields:

$$
\ln (1+\delta)+\delta \ln (1 / 2)>0
$$


And this last inequality is always true with strict inequality for $\delta \in] 0,1[$.

Second, we know that $g(0)=0$, so that $\lim _{r_{t} \rightarrow 0} \varphi=\lim _{r_{t} \rightarrow 0} \varphi_{g}=0$. Hence, we deduce that:

$$
\lim _{r_{t} \rightarrow 0}\left(\ln \left(x_{1}^{d e v}\left(x_{2}^{c}\left(r_{t}\right)\right)\right)+\delta V_{1, t+1}^{w}\left(r_{t+1}\right)\right)>\lim _{r_{t} \rightarrow 0}\left(V_{1, t}^{c}\left(r_{t}\right)\right)
$$

We can therefore deduce that there exists a strictly positive $r$ such that both players prefer deviating from the cooperative path and reverting to conflict $\forall r_{t} \leq$ $\underline{r}$.

\subsection{Proof of Proposition 2}

Proof. To establish this result we proceed in four steps. We first show (i) that when $r_{\tau} \rightarrow 0$, players strictly prefer declaring conflict in the first stage of the time period, instead of peacefully exploiting the resource in a non-cooperative manner. We then show (ii) that when $r_{\tau} \rightarrow \infty$, players strictly prefer foregoing immediate conflict and extracting non-cooperatively the resource, and (iii) that the utilities of the players being continuous in $r_{t}$ there exists values $\bar{r}_{\tau}$ and $\underline{r}_{\tau}$ such that $\forall r_{\tau}>\bar{r}_{\tau}$ players extract non-cooperatively the resource, and $\forall r_{\tau} \leq \underline{r}_{\tau}$ players declare conflict. Lastly, (iv) we show why the cooperative equilibrium is therefore not sustainable.

(i) Having shown that $\lim _{r_{\tau} \rightarrow 0} g\left(r_{\tau}\right)=0$, it follows that when resources tend to be fully depleted, under conflict the efficient solution can be fully replicated. By the definition of the efficient solution, we therefore have that when $r_{\tau} \rightarrow 0$, $\ln \left(x_{i}^{n c}\right)+\delta U_{i, \tau}^{w}\left(r_{\tau+1}\right)>V_{i, \tau}^{w}\left(r_{\tau}\right)$. 
(ii) We first re-express the implicit definition of $g\left(r_{\tau}\right)$ as given by (16) as:

$$
r_{\tau}=\frac{\left(4 g_{\tau}+\alpha\right)\left(1-\phi\left(g_{\tau}, g_{\tau}\right)\right)-2 g_{\tau}\left(2 g_{\tau}+\alpha\right) \phi_{g_{\tau}}}{\left(1-\phi\left(g_{\tau}, g_{\tau}\right)\right)-\left(2 g_{\tau}+\alpha\right) \phi_{g_{\tau}}}
$$

Taking $r_{\tau} \rightarrow \infty$, for the above equality to hold the RHS of the expression ought to tend to infinity as well. Assume first that $g_{-i}\left(r_{t}\right)$ tends to infinity, so that $\varphi\left(g_{i}, g_{-i}\right)=$ $1 \forall g_{i}$. Then by inspection of $(15)$, we deduce that $g_{i}\left(g_{j}\right)=0$. Notice next that $\forall g_{-i}$ s.t. $\phi\left(0, g_{-i}\right)<1, \exists$ a finite $\hat{g}_{i}$ s.t. $\phi\left(\hat{g}_{i}, g_{-i}\right)=1$ and $U_{i}\left(\hat{g}_{i}, g_{-i}\right)=-\infty$. Given that $\left.\forall g_{i} \in\right] 0, \hat{g}\left[, U_{i}\left(g_{i}, g_{-i}\right)>0, g_{i}\left(g_{-i}\right)<\hat{g}_{i}\right.$, and is therefore finite. If $g_{i}$ is finite, however, the above equality can only be true if the denominator tends to zero, which is verified when $\left(1-\phi\left(g_{i}\left(r_{\tau}\right), g_{j}\left(r_{\tau}\right)\right)\right)=\left(g_{i}\left(r_{\tau}\right)+g_{j}\left(r_{\tau}\right)+\alpha\right) \phi_{g_{i}\left(r_{\tau}\right)}$. The condition having to hold for both $i$ and $j$, it is necessary that $g_{i}=g_{j}$.

We then show that $\lim _{r_{\tau} \rightarrow \infty} V_{i, \tau}^{w}\left(r_{\tau}\right)<\lim _{r_{\tau} \rightarrow \infty} \ln \left(x_{i}^{n c}\left(r_{\tau}\right)\right)+\lim _{r_{\tau} \rightarrow \infty} \delta V_{i, \tau+1}^{w}\left(r_{\tau+1}\right)$. If in time period $\tau$, players do not declare conflict, and instead exploit the resource non-cooperatively, the maximization problem for player $i$ is given by:

$$
\max _{x_{i}} \ln \left(x_{i}\right)+\delta V_{i, \tau+1}^{w}\left(r_{\tau+1}\right)
$$

subject to $r_{\tau+1}=(1+\gamma)\left(1-x_{i, \tau}-x_{j, \tau}\right) r_{\tau}$. Optimizing for both players allows us to obtain: $x_{i}^{n c}\left(r_{\tau}\right)=x_{j}^{n c}\left(r_{\tau}\right)=\frac{1-\delta}{2-\delta} r_{\tau}$, and $r_{\tau+1}=\frac{(1+\gamma) \delta}{2-\delta} r_{\tau}$.

Using expression (17) and the values derived above, we can therefore write $V_{i, \tau}^{w}\left(r_{\tau}\right)-$ 
$\ln \left(x_{i}\right)-\delta V_{i, \tau}^{w}\left(r_{\tau+1}\right)<0$ as:

$$
\begin{array}{r}
\frac{1}{1-\delta} \ln \left(\frac{1-\delta}{2}\left(1-\varphi\left(g_{\tau}, g_{\tau}\right)\right)\left(r_{\tau}-2 g_{\tau}\right)\right)+\delta \ln \left(\frac{(1+\gamma) \delta}{(1-\delta)^{2}}\right) \\
-\ln \left(\frac{(1-\delta)}{2-\delta} r_{\tau}\right)-\frac{\delta}{1-\delta} \ln \left(\frac{1-\delta}{2}\left(1-\varphi\left(g_{\tau+1}, g_{\tau+1}\right)\right)\left(\frac{(1+\gamma) \delta}{2-\delta} r_{\tau}-2 g_{\tau+1}\right)\right) \\
-\frac{\delta^{2}}{(1-\delta)^{2}} \ln ((1+\gamma) \delta)<0
\end{array}
$$

We next collect terms ans simplify the above expression, take the limit of the condition as $r_{\tau} \rightarrow \infty$, use the fact that then $g_{\tau}=g_{\tau+1}$, and we then obtain:

$\ln \left(\frac{1-\phi\left(g_{\tau}, g_{\tau}\right)}{2}\right)+\frac{\delta}{1-\delta} \ln ((1+\gamma) \delta)+\ln (2-\delta)<\lim _{r_{\tau} \rightarrow \infty} \ln \left(\frac{r_{\tau}\left[\frac{(1+\gamma) \delta r_{\tau}}{2-\delta}-2 g_{\tau}\right]}{r_{\tau}-2 g_{\tau}}\right)$

Applying l'Hôspital's rule to the bracketed term of the RHS of this expression, we deduce that the bracketed term tends to infinity given that:

$$
\lim _{r_{\tau} \rightarrow \infty} \frac{\frac{2(1+\gamma) \delta r_{\tau}}{2-\delta}-2 g_{\tau}}{1}=\infty
$$

As a consequence, when $r_{\tau} \rightarrow \infty, \ln \left(x_{i}^{n c}\right)+\delta V_{i, \tau}^{w}\left(r_{\tau+1}\right)>V_{i, \tau}^{w}\left(r_{\tau}\right)$, and players exploit the resource non-cooperatively in time $\tau$.

(iii) The players' utility being continuous in the level of resources, by the intermediate values theorem the threshold values $\bar{r}$ and $\underline{r}$ must exist.

(iv) Points (i) to (iii) enable us to conclude that there exists a $\underline{r}$ such that conflict occurs. For any $r \leq \underline{r}$ cooperation cannot be sustained by a punishment 
threat given that otherwise in the punishment period's first stage the punished player would declare conflict. Can cooperation then be sustained for $r_{\tau}>\underline{r}$ ? The worst punishment that can be imposed gives to the punished player the same utility as the conflict strategy for the same reason as above. Assume that in $\tau, r_{\tau}=\underline{r}+\epsilon$. If player 2 deviates in $\tau-1$, denote by $\hat{x}_{2}$ the punishment action of player 2 in $\tau$, and by $\hat{x}_{1}$ the action of player 1 . If player 1 is better-off by extracting $\hat{x}_{1}$ than $x^{\operatorname{dev}}\left(\hat{x}_{2}\right)$, it is necessarily the case that player 2 is better off by playing non-cooperatively instead of accepting the punishment, that is: $\ln \left(x^{\operatorname{dev}}\left(\hat{x}_{1}\right)\right)+\delta V_{\tau+1}^{w}\left((1+\gamma)\left(r_{\tau}-\hat{x}_{1}-x^{\operatorname{dev}}\left(\hat{x}_{1}\right)\right)\right)>$ $\ln \left(\hat{x}_{2}\right)+\delta V_{\tau+1}^{w}\left((1+\gamma)\left(r_{\tau}-\hat{x}_{1}-\hat{x}_{2}\right)\right)$. If player 1 is worse-off by extracting $\hat{x}_{1}$ than $x^{\text {dev }}\left(\hat{x}_{2}\right)$, player 1 has incentives to deviate from the punishment scheme. In time period $\tau$, the punishment is therefore not credible, and in $\tau-1$, cooperation is not sustainable. Applying the argument backwardly completes the proof. 


\section{Bibliography}

Acemoglu, Daron, Mikhail Golosov, Aleh Tsyvinski, and Pierre Yared. 2012. "A Dynamic Theory of Resource Wars." Quartely Journal of Economics 127:283331.

Baland, Jean-Marie, and Jean-Philippe Platteau. 1996. Halting Degradation of Natural Resources: Is There a Role for Local Communities? Food and Agriculture Organization of the United Nations.

Bester, Helmut, and Kai Konrad. 2004. "Delay in Contests." European Economic Review 48:1169-1178.

Cave, Jonathan. 1987. "Long-Term Competition in a Dynamic Game: the Cold Fish War." RAND Journal of Economics 18 (4):596-610.

Chassang, Sylvain, and Gerard Padró i Miquel. 2009. "Conflict and Deterrence Under Strategic Risk." Quartely Journal of Economics, vol. forthcoming.

Coase, Ronald. 1960. "The Problem of Social Cost." Journal of Law and Economics $3(1): 1-44$. 
Diamond, Jared M. 2005. Collapse: How Societies Choose to Fail or Succeed. Viking Press.

Dutta, Prajit K. 1995. "A Folk Theorem for Stochastic Games." Journal of Economic Theory 66:1-32.

Dutta, Prajit K., and Roy Radner. 2009. "A Strategic Analysis of Global Warming: Theory and Some Numbers." Journal of Economic Behavior \&3 Organization 71:187-209.

Esteban, Joan, Massimo Morelli, and Dominic Rohner. 2011. "Strategic Mass Killings." Barcelona Economics Working Paper Series, vol. WP 459.

Garfinkel, Michelle. 1990. "Arming as a Strategic Investment in a Cooperative Equilibrium." American Economic Review 80 (1):50-68.

Hardin, Garrett. 1968. "The Tragedy of the Commons." Science 162:1243-1248.

Hunt, Terry, and Carl Lipo. 2011. The Statues that Walked: Unravelling the Mystery of Easter Island. Free Press, New-York.

Jackson, Matthew O., and Massimo Morelli. 2009. "Strategic Militarization, Deterrence and Wars." Quartely Journal of Political Science 4 (4):279-313.

Leventoglu, Bahar, and Branislav L. Slantchev. 2007. "The Armed Peace: A Punctuated Equilibrium Theory of War." American Journal of Political Science $51(4): 755-771$.

Levhari, David, and Leonard J Mirman. 1980. "The Great Fish War: an Example Using a Dynamic Cournot-Nash Solution." The Bell Journal of Economics 11 (1):322-334. 
McBride, Michael, and Stergios Skaperdas. 2007. Chapter Explaining Conflict in Low-Income Countries: Incomplete Contracting in the Shadow of the Future of Institutions and Norms in Economic Development, edited by M. Gradstein and (eds.) K.A. Konrad, 141-161. Cambridge MA: MIT Press.

Ostrom, Elinor. 1990. Governing the Commons: the Evolution of Institutions for Collective Action. Cambridge University Press, Cambridge.

Powell, Robert. 2012. "Persisting Conflict and Shifting Power." American Journal of Political Science 56 (3):620-637.

Sorger, Gerhard. 2005. "A dynamic Common Property Resource Problem With Amenity Value and Extraction Costs." International Journal of Economic Theory $1(1): 3-19$.

Yared, Pierre. 2010. "A dynamic theory of war and peace." Journal of Economic Theory 145:1921-1950. 